(c) 2003 International Press

Adv. Theor. Math. Phys. 7 (2003) 711-725

\title{
Higher-level eigenvalues of Q-operators and Schrödinger equation
}

\author{
V.V. Bazhanov ${ }^{1}$, S.L. Lukyanov ${ }^{2,3}$ and A.B. Zamolodchikov ${ }^{2,3}$ \\ ${ }^{1}$ Department of Theoretical Physics, \\ Research School of Physical Sciences and Engineering, \\ IAS, Australian National University, \\ Canberra, ACT 0200, Australia \\ ${ }^{2}$ NHETC, Department of Physics and Astronomy \\ Rutgers University \\ Piscataway, NJ 08855-0849, USA \\ ${ }^{3}$ L.D. Landau Institute for Theoretical Physics \\ Chernogolovka, 142432, Russia
}

\begin{abstract}
Relation between one-dimensional Schrödinger equation and the vacuum eigenvalues of the $\mathbf{Q}$-operators is extended to their higher-level eigenvalues.
\end{abstract}

e-print archive: http://lanl.arXiv.org/abs/hep-th/0307108 


\section{Introduction}

Remarkable relation between the spectral theory of Schrödinger equation [1] and vacuum eigenvalues of the so-called $\mathbf{Q}$ operators of Conformal Field Theory (CFT) was discovered a few years ago in [2]. The Q-operators were introduced in [3] as a mathematical tool to describe integrable (Yang-Baxter) structure of $c \leq 1 \mathrm{CFT}$, where they play the role of the Baxter's $Q$-matrix [4]. It was observed in [2] that for several degenerate Virasoro module the vacuum eigenvalues of the $\mathbf{Q}$-operators essentially coincide with the spectral determinants of the Schrödinger operator $-\frac{\mathrm{d}^{2}}{\mathrm{~d} x^{2}}+V(x)$ with the potential $V(x)=x^{2 \alpha}$, where the exponent $\alpha$ is determined by the Virasoro central charge $c$. This relation was extended in [5] to all Virasoro modules with $c<1$. On the other hand, the Virasoro vacuum is one among a sequence of eigenstates of the operators $\mathbf{Q}$ in the whole Virasoro module. The question arises if such relation to the Schrödinger operator exists for the higher-level eigenvalues.

In this note we answer this question positively and identify the Schrödinger operators associated with the higher-level eigenvalues. For an eigenstate on the level $L$ of the highest-weight Virasoro module the potential has the form

$$
V(x)=\frac{\ell(\ell+1)}{x^{2}}+x^{2 \alpha}-2 \frac{\mathrm{d}^{2}}{\mathrm{~d} x^{2}} \sum_{k=1}^{L} \log \left(x^{2 \alpha+2}-z_{k}\right),
$$

where $\alpha$ and $\ell$ are related to the central charge $c$ and the highest weight $\Delta$ of the Virasoro module as follows

$$
c=1-\frac{6 \alpha^{2}}{\alpha+1}, \quad \Delta=\frac{(2 \ell+1)^{2}-4 \alpha^{2}}{16(\alpha+1)} .
$$

The $z_{k}, k=1,2, \ldots L$ are pairwise different nonzero complex numbers satisfying the system of $L$ algebraic equations

$$
\sum_{\substack{j=1 \\ j \neq k}}^{L} \frac{z_{k}\left(z_{k}^{2}+(3+\alpha)(1+2 \alpha) z_{k} z_{j}+\alpha(1+2 \alpha) z_{j}^{2}\right)}{\left(z_{k}-z_{j}\right)^{3}}-\frac{\alpha z_{k}}{4(1+\alpha)}+\Delta=0
$$

with $k=1,2, \ldots L$. In what follows we refer to the potentials (1) as the "Qpotentials". The arguments presented below strongly suggest that appropriately defined spectral determinants of the Schrödinger operator $-\frac{\mathrm{d}^{2}}{\mathrm{~d} x^{2}}+V(x)$ with the Q-potentials $V(x)$ coincide, up to certain trivial factors, with the eigenvalues of the Q-operators.

The Schrödinger equation with the monstrous potentials (1) are unlikely to be of much direct interest in quantum mechanics. On the other hand, 
the higher-level eigenvalues of the Q-operators are definitely of interest in integrable quantum field theories. One of the reasons is their relation $[3,6]$ to the boundary states associated with certain integrable boundary flows $[7,8]$. This work was motivated by possible applications to this kind of problems.

We did not attempt at making this paper self-contained. The definitions of the Q-operators and the local and nonlocal integrals of motion are as in Ref. [3]. The arguments in Section 3 are meant to be read in conjunction with Refs. $[2,5]$.

\section{The Q-operators}

Here we only list some basic properties of the Q-operators used in the discussion below. We refer the reader to $[3,9]$ for explicit construction of the Q-operators and more details.

The $\mathbf{Q}$-operators are actually the operator-valued functions $\mathbf{Q}_{ \pm}(s)$ of a complex parameter $s^{1}$. The operators $\mathbf{Q}_{ \pm}(s)$ act in the Virasoro module $\mathcal{V}_{\Delta}$. It will be convenient to parametrize its highest weight $\Delta$ and the Virasoro central charge $c<1$ as in Eqs.(2), where we assume that the real parameters $\ell$ and $\alpha$ are in the range $\ell \geq-1 / 2, \alpha>0$. The operators $\mathbf{Q}_{ \pm}(s)$ form a commutative family and they also commute with the zero-mode Virasoro generator $\mathbf{L}_{0}$. The Virasoro module $\mathcal{V}_{\Delta}$ admits the standard level decomposition,

$$
\mathcal{V}_{\Delta}=\oplus_{L=0}^{\infty} \mathcal{V}_{\Delta}^{(L)}, \quad \mathbf{L}_{0} \mathcal{V}_{\Delta}^{(L)}=(\Delta+L) \mathcal{V}_{\Delta}^{(L)},
$$

and the level subspaces $\mathcal{V}_{\Delta}^{(L)}$ are invariant with respect to the action of the Q-operators,

$$
\mathbf{Q}_{ \pm}: \mathcal{V}_{\Delta}^{(L)} \rightarrow \mathcal{V}_{\Delta}^{(L)}
$$

In particular, the Virasoro vacuum $|\Delta\rangle$ is an eigenvector of the Q-operators,

$$
\mathbf{Q}_{ \pm}(s)|\Delta\rangle=Q_{ \pm}^{(v a c)}(s)|\Delta\rangle
$$

It is the vacuum eigenvalues $Q_{ \pm}^{(v a c)}(s)$ that have received most of attention in the Refs. $[2,5]$. Here we will discuss generic eigenvalues $Q_{ \pm}(s)$ of the

\footnotetext{
${ }^{1}$ The operators $\mathbf{Q}_{ \pm}(s)$ discussed here coincide with $\mathbf{Q}_{ \pm}(\lambda)$ of $[3,9]$ with

$$
\lambda^{2}=\left[\sqrt{\pi} \frac{\Gamma\left(\frac{1+\alpha}{2 \alpha}\right)}{\Gamma\left(\frac{1}{2 \alpha}\right)}\right]^{\frac{2 \alpha}{1+\alpha}} \Gamma^{-2}\left(\frac{\alpha}{1+\alpha}\right) s .
$$

The parameter $\alpha$ is related to $\beta$ used in $[3,9]$ as $\beta^{2}=\frac{1}{1+\alpha}$.
} 
operators $\mathbf{Q}_{ \pm}(s)$ in $\mathcal{V}_{\Delta}$. As the functions of $s$ they enjoy the following basic properties $[3,9]^{2}$;

(i) The functions $A_{ \pm}(s)=(-s)^{\mp \frac{2 \ell+1}{4}} Q_{ \pm}(s)$ are entire functions of $s$, and

$$
A_{ \pm}(0)=1 \text {. }
$$

(ii) The eigenvalues $Q_{ \pm}(s)$ have the leading asymptotic behavior:

$$
\log Q_{ \pm}(s)=\frac{\pi}{\cos \left(\frac{\pi}{2 \alpha}\right)}(-s)^{\frac{1+\alpha}{2 \alpha}}+O(1)
$$

at $|s| \rightarrow \infty, \arg (-s)<\pi$.

(iii) The functions $A_{ \pm}(s)$ satisfy the functional relation (the "quantum Wronskian condition")

$$
q^{\ell+\frac{1}{2}} A_{+}(q s) A_{-}\left(q^{-1} s\right)-q^{-\ell-\frac{1}{2}} A_{+}\left(q^{-1} s\right) A_{-}(q s)=q^{\ell+\frac{1}{2}}-q^{-\ell-\frac{1}{2}},
$$

where

$$
q=\mathrm{e}^{\frac{\mathrm{i} \pi}{1+\alpha}}
$$

The equation (9) follows directly from the construction of the Q-operators $[3,9]$. The statements of analyticity, $(i)$ and (ii) above, and especially the domain of validity of the asymptotic form (8), presently have somewhat less solid status. For the vacuum eigenvalues $Q_{ \pm}^{(v a c)}(s)$ these properties follow from the results of [5]. The solution at $c=-2$, where all eigenvalues can be found explicitly [3], also supports the above analyticity statement $(i),(i i)$ (see footnote ${ }^{2}$ on this page). But it is fair to say that a solid proof in a general case is still missing. Nonetheless, in the further discussion we assume these properties.

Entire functions $A_{ \pm}(s)$ having the asymptotic behavior (8) are characterized by positions of their respective zeros $s_{k}^{ \pm}$in the complex $s$-plane. The zeros accumulate towards $s=\infty$ along the positive real axis. The quantum Wronskian equation (9) then leads to infinite set of the Bethe Ansatz equations for the numbers $s_{k}^{ \pm}$. The analysis of these equations in Appendix A shows that there is a discrete (albeit infinite) set of functions $Q_{ \pm}(s)$ that satisfy all the conditions $(i-i i i)$ above. Clearly, all eigenvalues of the Qoperators can be found among this set. But there is much evidence that the

\footnotetext{
${ }^{2}$ In writing Eqs. (8),(9) below we assume that $(1+\alpha) /(2 \alpha)$ is not an integer. If it equals to $N=1,2, \ldots$, the asymptotic form (8) changes to $s^{N} \log (-s)$ and (9) must be modified accordingly. The free-fermion point $\alpha=1$ (i.e. $c=-2$ ) is one of these "resonance" cases.
} 
converse is also true - any pair of functions $Q_{ \pm}(s)$ satisfying $(i-i i i)$ are eigenvalues of the pair of operators $\mathbf{Q}_{ \pm}(s)$ in $\mathcal{V}_{\Delta}$. Given a solution of $(i-i i i)$, the level $L$ of the associated eigenvector can be identified as follows. Corrections to the leading asymptotics (8) are expressed through the eigenvalues of the local and nonlocal integrals of motion, according to the expansion (26) below. Since the first local integral $\mathbf{I}_{1}$ coincides with the Virasoro generator $\mathbf{L}_{0}-c / 24$, the first subleading terms to the asymptotics (8) have the form $(\alpha>1 / 2)$ :

$$
\begin{aligned}
\log Q_{ \pm}(s) \simeq & \frac{\pi}{\cos \left(\frac{\pi}{2 \alpha}\right)}(-s)^{\frac{1+\alpha}{2 \alpha}}+C \\
& -\frac{1}{\sin \left(\frac{\pi}{2 \alpha}\right)}\left(\Delta-\frac{c}{24}+L\right)(-s)^{-\frac{1+\alpha}{2 \alpha}}+\ldots
\end{aligned}
$$

where the constant term does not depend on $s$ (but, of course, depends on a state from $V_{\Delta}$ ) and $L$ is the level. The results of Appendix A suggest that for generic $c$ and $\Delta$ the number of those solutions of $(i-i i i)$ which also have the subleading behavior (11) with a given integer $L \geq 0$ equals the number of integer partitions of $L$. It thus coincides with the dimension of the level subspace $\mathcal{V}_{\Delta}^{(L)}$ of a generic non-degenerate Virasoro module $\mathcal{V}_{\Delta}$.

\section{Schrödinger equation}

It is known (see e.g. $[1,5]$ ) that appropriately defined spectral determinants $D_{ \pm}(E)$ (Eq.(21) below) for the Schrödinger equation

$$
-\frac{\mathrm{d}^{2}}{\mathrm{~d} x^{2}} \Psi(x)+V(x) \Psi(x)=E \Psi(x)
$$

with the potential

$$
V(x)=V^{(v a c)}(x) \equiv \frac{\ell(\ell+1)}{x^{2}}+x^{2 \alpha}
$$

obey all the above properties $(i-i i i)$, if one makes the identifications

$$
D_{ \pm}(\nu s)=A_{ \pm}(s)
$$

where

$$
\nu=\left[\frac{2 \sqrt{\pi} \Gamma\left(\frac{3}{2}+\frac{1}{2 \alpha}\right)}{\Gamma\left(1+\frac{1}{2 \alpha}\right)}\right]^{\frac{2 \alpha}{1+\alpha}} .
$$

Thus $D_{ \pm}(E)$ are related to some eigenvalues of the Q-operators. In fact, the spectral determinants of (12) with $V(x)=V^{(v a c)}(x)$ correspond to the 
vacuum eigenvalues $Q_{ \pm}^{(v a c)}$. This correspondence in the case $\ell=0$ is the original observation of [2]. It was proven in [5] by combining Eq.(9) with known analyticity of $Q_{ \pm}^{(v a c)}$ as functions of the parameter $\ell$.

It is important that the properties $(i-i i i)$ of the spectral determinants can be derived using only the following three properties of the potential (13) as the function of complex $x$ :

- (I) Symmetry:

$$
V(q x)=q^{-2} V(x),
$$

where $q$ is defined in (10).

- (II) Asymptotic behavior:

$$
\begin{array}{ll}
V(x) \rightarrow \frac{\ell(\ell+1)}{x^{2}}+o(1) & \text { as } \quad x \rightarrow 0, \\
V(x) \rightarrow x^{2 \alpha}+o(1) & \text { as } \quad x \rightarrow \infty .
\end{array}
$$

- (III) Monodromy properties: For any value of $E$ all solutions of (12) with this potential are monodromy free everywhere except for $x=0$ and $x=\infty$.

Here we observe that $V^{(v a c)}(x)$ is not the only potential which meets all of the conditions $(I-I I I)$. We show in Appendix B that the most general potential $V(x)$ with these properties is given by (1), with the parameters $z_{k}$ satisfying the Eqs.(3). Note that for $L>0$ the potential (1) has poles away from zero, at $x^{2 \alpha+2}=z_{1}, z_{2}, \ldots, z_{L}$. The equations (3) then guarantee that these poles do not violate $(I I I)$. Thus $(I-I I I)$ are the characteristic properties of all Q-potentials.

Given a Q-potential, Eqs.(1),(3), the associated spectral determinants $D_{ \pm}(E)$ are defined as follows. For $\Re e \ell>(1+\alpha) L-3 / 2$ the equation (12) has a solution $\psi(x, \ell, E)$ uniquely specified by the condition

$$
\psi(x, E, \ell): \quad \psi(x, E, \ell)=x^{\ell+1}+O\left(x^{\ell+3}\right) \quad \text { as } \quad x \rightarrow 0 .
$$

This solution can be analytically continued outside the domain $\Re e \ell>(1+$ a) $L-3 / 2$. Let $\psi(x, E,-\ell-1)$ be another solution of the same equation (12) obtained from $\psi(x, E, \ell)$ by appropriate analytic continuation in $\ell^{3}$.

\footnotetext{
${ }^{3}$ In general, analytic continuation of $\psi(x, E, \ell)$ in $\ell$ yields a multivalued function of $\ell$, and $\psi(x, E,-\ell-1)$ at different branches solves (12) with different potentials, corresponding to different solutions of the same equations (3). However, since $\ell$ enters the Eq.(3) only through $\Delta$, Eq.(2), there exists a continuation $\ell \rightarrow-\ell-1$ which leaves the potential (1) unchanged.
} 
For generic values of $\ell$ the solutions $\psi_{+}(x, E)=\psi(x, E, \ell)$ and $\psi_{-}(x, E)=$ $\psi^{+}(x, E,-\ell-1)$ are linearly independent, since their Wronskian

$$
W\left[\psi_{+}, \psi_{-}\right]=\psi_{+} \partial_{x} \psi_{-}-\psi_{-} \partial_{x} \psi_{+}
$$

equals to $-(2 \ell+1)$.

For certain isolated values of $E$ one of these solutions decays at $x \rightarrow$ $+\infty$. Let $\left\{E_{k}^{+}\right\}_{k=1}^{\infty}$ and $\left\{E_{k}^{-}\right\}_{k=1}^{\infty}$ be ordered spectral sets defined by the conditions

$$
\psi_{k}^{+}(x) \equiv \psi^{+}\left(x, E_{k}^{+}\right) \rightarrow 0, \quad \psi_{k}^{-}(x) \equiv \psi^{-}\left(x, E_{k}^{-}\right) \rightarrow 0,
$$

as $x \rightarrow+\infty$. One then defines

$$
D_{ \pm}(E)=\prod_{k=1}^{\infty}\left(1-\frac{E}{E_{k}^{ \pm}}\right) .
$$

Straightforward WKB analysis of the equation (12) shows that

$$
E_{k}^{ \pm}=\nu\left(k-\frac{1}{2} \pm \frac{2 l+1}{4}\right)^{\frac{2 \alpha}{1+\alpha}}(1+o(1)), \quad \text { as } \quad k \rightarrow \infty,
$$

and therefore at $\alpha>1$ these products converge for all finite $E$. For $0<\alpha \leq 1$ the definition (21) has to be modified by including the Weierstrass factors, but this does not affect our arguments.

The spectral determinant $D_{ \pm}(E)$ thus defined are entire functions of $E$. Asymptotic behavior of these functions at large $E$ follows from (22),

$$
\log D_{ \pm}(E)=(-E / \nu)^{\frac{1+\alpha}{2 \alpha}} \mp \frac{2 l+1}{4} \log (-E)+O(1), \quad \arg (-E)<\pi .
$$

And one can repeat the arguments of [5] to show that the spectral determinants (21) satisfy exactly the same functional relation (9), as the direct consequence of the properties $(I-I I I)$ of the Q-potential. We conclude that the pair of functions $D_{ \pm}(E)$ satisfies, upon the identifications (14), all the analytic conditions $(i-i i i)$ of Section 2 . This shows that every Q-potential (1) corresponds to some eigenvector of the Q-operators, and the associated eigenvalues $Q_{ \pm}(s)$ coincide, up to the factors $(-s)^{ \pm \frac{2 \ell+1}{4}}$, with the spectral determinants $D_{ \pm}(\nu s)$. Moreover, using the WKB expansion of the solutions of (12), it is possible to evaluate corrections to the leading asymptotics (23) for any Q-potential (1). For the first such correction one obtains exactly (11) with $L$ standing for the number of nonzero poles $z_{1}, z_{2}, \ldots, z_{L}$ of (1). This confirms the identification of $L$ in (1) with the level.

At this point the question arises: is this description of the spectrum of the Q-operators complete? In other words, is the family of potentials 
(1) large enough to accommodate all the eigenvalues $Q_{ \pm}(s)$ ? Answering this question requires enumerating all solutions of the algebraic equations (3) for an arbitrary level $L$. We did not accomplish this task. However, an elementary analysis of a few lowest levels suggests positive answer to this question. For instance, for $\Delta \neq 0$ the level 1 subspace $\mathcal{V}_{\Delta}^{(1)}$ is onedimensional. Correspondingly, for $L=1$ the equation (3) has only one solution

$$
z_{1}=z=\frac{4(1+\alpha)}{\alpha} \Delta
$$

Therefore the spectral determinants $D_{ \pm}(E)$ of (12) with

$$
\begin{aligned}
V(x)=x^{-2}\left\{x^{2 \alpha+2}+\ell(\ell+1)+4 \alpha+4\right. & \\
& \left.\quad+\frac{4(\alpha+1)(2 \alpha+3) z}{x^{2 \alpha+2}-z}+\frac{8(\alpha+1)^{2} z^{2}}{\left(x^{2 \alpha+2}-z\right)^{2}}\right\}
\end{aligned}
$$

coincide with the eigenvalues of $\mathbf{Q}_{ \pm}(s)$ corresponding to the eigenvector $\mathbf{L}_{-1}|\Delta\rangle$. Note that $z$ turns to zero at $\Delta=0$, in agreement with the fact that in this case $\mathbf{L}_{-1}|\Delta\rangle$ becomes a null-vector.

For $L=2$ the system (3) is solved by elementary methods. For generic $c$ and $\Delta$ it has exactly two solutions (of course we do not distinguish solutions which differ by permutations of $z_{k}$ ). We can recall that for a non-degenerate Virasoro module the dimension of $\mathcal{V}_{\Delta}^{(2)}$ equals 2. Again, one of the solutions disappears (in the sense that one or both of the numbers $z_{1}, z_{2}$ turn to zero) in three cases $\Delta=0, \Delta=\frac{1-2 \alpha}{4(1+\alpha)}$ and $\Delta=\frac{1+3 \alpha}{4}$, as expected, because at these values of $\Delta$ null-vectors appear at the level 1 or 2 , and the dimension of $\mathcal{V}_{\Delta}^{(2)}$ drops down. Similar situation is observed at the level 3.

\section{Local integrals of motion}

Finally, let us make remark on the eigenvalues of the local Integrals of Motion (IM) and their relation to the semiclassical analysis of the Schrödinger equation with the Q-potentials (1). Asymptotic expansions of the operators $\mathbf{Q}_{ \pm}(s)$ around the essential singularity at $s=\infty$ (in the sector $-\pi<$ $\arg (-s)<\pi)$ are expressed in terms of the infinite sequence of the local IM 
$\mathbf{I}_{2 n-1}$ and "dual nonlocal IM" $\widetilde{\mathbf{H}}_{n}^{ \pm}[3]$,

$$
\begin{aligned}
\log \mathbf{Q}_{ \pm}(s) \simeq & \frac{\pi}{\cos \left(\frac{\pi}{2 \alpha}\right)}(-s)^{\frac{1+\alpha}{2 \alpha}}+\mathbf{C}+\sum_{n=1}^{\infty}(-\nu s)^{\frac{(1-2 n)(1+\alpha)}{2 \alpha}} b_{n} \mathbf{I}_{2 n-1} \\
& +\sum_{n=1}^{\infty}(-\nu s)^{-n(1+\alpha)} c_{n} \widetilde{\mathbf{H}}_{n}^{ \pm}
\end{aligned}
$$

where the coefficients $b_{n}$ and $c_{n}$ have explicit form

$$
\begin{aligned}
& b_{n}=\frac{(-4)^{n-1}}{n ! \alpha \sqrt{\pi}}(1+\alpha)^{n} \Gamma\left(\frac{1-2 n}{2 \alpha}\right) \Gamma\left(\frac{(2 n-1)(1+\alpha)}{2 \alpha}\right) \\
& c_{n}=(-1)^{n-1} 4^{n \alpha}(1+\alpha)^{-2 n}
\end{aligned}
$$

The asymptotic expansions of the eigenvalues $Q_{ \pm}(s)$ are thus expressed through the eigenvalues of the local and nonlocal IM. Note that (26) has exactly the structure which appears when one performs the WKB expansion of the solutions of the Schrödinger equation (12) with a Q-potential (1). The correspondence (14) suggested in the previous section implies that the coefficients generated by this WKB expansion must agree with the eigenvalues of the operators $\mathbf{I}_{2 n-1}$ and $\widetilde{\mathbf{H}}_{n}^{ \pm}$.

Whereas the eigenvalues of the dual nonlocal IM are relatively difficult (but not impossible) to calculate, the local IM $\mathbf{I}_{2 n-1}$ have explicit expressions in terms of the Virasoro generators $\mathbf{L}_{n}$ (see Eq.(11) of Ref. [6]), and their eigenvalues in any given level subspace $\mathcal{V}_{\Delta}^{(L)}$ can be found by a direct calculation. Thus, for the vacuum eigenvalues of the first few $\mathbf{I}_{2 n-1}$ we have [6]

$$
\begin{aligned}
I_{1}^{(v a c)}(\Delta)= & \Delta-\frac{c}{24}, \\
I_{3}^{(v a c)}(\Delta)= & \Delta^{2}-\frac{c+2}{12} \Delta+\frac{c(5 c+22)}{2880}, \\
I_{5}^{(v a c)}(\Delta)= & \Delta^{3}-\frac{c+4}{8} \Delta^{2}+\frac{(c+2)(3 c+20)}{576} \Delta \\
& -\frac{c(3 c+14)(7 c+68)}{290304},
\end{aligned}
$$

where the Virasoro central charge $c$ is related to the parameter $\alpha$ as in Eq.(2). We have checked by explicit diagonalization at the levels $L=1,2,3$ that the 
eigenvalues of the first three local IM agree with the following expressions

$$
\begin{aligned}
I_{1}= & I_{1}^{(v a c)}(\Delta+L) \\
I_{3}= & I_{3}^{(v a c)}(\Delta+L)+\frac{\alpha}{1+\alpha} \sum_{k=1}^{L} z_{k} \\
I_{5}= & I_{5}^{(v a c)}(\Delta+L)+\left(\frac{\Delta+L-1}{5+2 \alpha}+\frac{3+\alpha}{24(1+\alpha)}\right) \times \\
& \frac{3 \alpha(3+2 \alpha)}{1+\alpha} \sum_{k=1}^{L} z_{k}+\frac{3 \alpha^{2}}{2(5+2 \alpha)(1+\alpha)^{2}} \sum_{k=1}^{L} z_{k}^{2},
\end{aligned}
$$

in terms of the same parameters $z_{k}$ that enter (1). One can verify that the same expressions come out from the WKB analysis of Schrödinger equation $(12),(1)$.

\section{Acknowledgments}

The authors thank C. Ahn for reading the manuscript and important remarks. VVB thanks the Department of Physics and Astronomy at Rutgers University for the hospitality extended to him during his visit to Rutgers in April 1999 when most of the results of this paper have been obtained. He also thanks A.N. Kirillov for interesting discussions.

The research of SLL and ABZ is supported in part by DOE grant \#DEFG02-96 ER 40949.

\section{Appendix A}

In this Appendix we assume that $\alpha$ and $\ell$ take some generic values so that the Virasoro module $\mathcal{V}_{\Delta}$ is non-degenerate. Moreover we assume that $\alpha>1^{4}$. Then the entire functions $A_{ \pm}(s)=(-s)^{\mp \frac{2 \ell+1}{4}} Q_{ \pm}(s)$ are uniquely determined by their leading asymptotics at $s \rightarrow \infty$ (8), the normalization (7), and the locations of their respective zeroes $s_{k},(k=1,2, \ldots)$ in the complex $s$-plane.

\footnotetext{
${ }^{4}$ For $0<\alpha \leq 1$ the definition (A.3),(A.4) below may need modification, but the statement about the one to one correspondence between the eigenstates and the sets (A.6),(A.7) most likely remains valid.
} 
From the quantum Wronskian relation (9) it follows that for any eigenvector of $\mathbf{Q}_{ \pm}(s)$ the zeroes $s_{k}^{ \pm}$of $A_{ \pm}(s)$ satisfy the same infinite system of the Bethe Ansatz equations

$$
\frac{A_{ \pm}\left(s_{k}^{ \pm} q^{2}\right)}{A_{ \pm}\left(s_{k}^{ \pm} q^{-2}\right)}=-q^{\mp(2 \ell+1)} .
$$

The eigenvalues corresponding to different eigenvectors are distinguished by a specific phase assignment in this equation

$$
\frac{1}{2 \pi \mathrm{i}} \log \left[\frac{A_{ \pm}\left(s_{k}^{ \pm} q^{2}\right)}{A_{ \pm}\left(s_{k}^{ \pm} q^{-2}\right)}\right]=\mp \frac{2 \ell+1}{2(1+\alpha)}-m_{k}^{ \pm}+\frac{1}{2}
$$

determined by a choice of integers $\left\{m_{k}^{ \pm}\right\}_{k=1}^{\infty}$. These integers, of course, depend on the choice of branches of the logarithm in the left hand side of (A.2). However, once these branches are appropriately fixed (see below), every eigenvalue is characterized by a unique set of the integers $\left\{m_{k}^{ \pm}\right\}$.

As follows from $(i i)$ in the Section 2, the zeroes $s_{k}^{ \pm}$accumulate toward infinity along the positive real axis. In fact, explicit solutions for special values of $c$, as well as numerical analysis, give all support to the following

Conjecture: For all eigenvalues at the level $L$, and for real $\ell>(1+$ $\alpha) L-3 / 2$ all zeroes $s_{k}^{+}$of $A_{+}(s)$ are simple, real and positive. The zeroes of $A_{-}(s)$ enjoy the same property for $\ell<-(1+\alpha) L+3 / 2$.

Assuming this property one can unambiguously fix the phase of the logarithm in (A.2). Consider, for example, the zeroes $\left\{s_{k}^{+}\right\}$of $A_{+}(s)$ for $\ell>(1+\alpha) L-3 / 2$ and define the L.H.S. of Eq.(A.2) as

$$
\text { L.H.S. of Eq.(A.2) }=\frac{1}{\pi} \sum_{j=1}^{\infty} \arg \left(1-q^{2} s_{k} / s_{j}\right) \text {, }
$$

where for real positive $x$

$$
-\pi<\arg \left(1-q^{2} x\right)<+\pi .
$$

Since all zeroes $s_{k}^{+}$are assumed to be distinct and positive they can be uniquely ordered by their value $s_{1}^{+}<s_{2}^{+}<s_{3}^{+}<\ldots$. Then for each eigenvalue $A_{+}(s)$ all the integers $m_{k}^{+}$will be distinct and uniquely defined. For other values of $\ell$ the L.H.S. of (A.2) is defined by means of the analytic continuation of (A.3) in $\ell$. 
Obviously, not every set of integers $\left\{m_{k}^{ \pm}\right\}$corresponds to an eigenstate of $\mathbf{Q}_{ \pm}(s)$ in $\mathcal{V}_{\Delta}$. Indeed, it follows from (8) that

$$
s_{k}^{ \pm}=\left(k-\frac{1}{2} \pm \frac{2 l+1}{4}\right)^{\frac{2 \alpha}{1+\alpha}}(1+o(1)), \quad \text { as } \quad k \rightarrow \infty .
$$

Hence (A.2) implies that for a given eigenvalue the sequence of integers $m_{k}^{ \pm}$ stabilize at large $k$, i.e. $m_{k}^{ \pm}=k$ for sufficiently large $k$. The sets $\left\{m_{k}^{ \pm}\right\}$associated with different eigenvalues differ in finitely many first entries. Therefore the most general pattern in the set $\left\{m_{k}^{+}\right\}$(or $\left\{m_{k}^{-}\right\}$) can be obtained from the vacuum set $\left(m_{k}^{+}=k\right.$, for all $\left.k=1,2, \ldots, \infty\right)$ by deleting a certain number of entries (we denote this number by $M$ ) and adding the same number of distinct non-positive integer entries. It can be written as

$$
m_{k}^{+}=\left\{\begin{array}{cl}
1-n_{M-k+1}^{-}, & \text {for } k=1, \ldots M \\
N_{k-M}\left(n^{+}\right), & \text {for } k \geq M+1
\end{array}\right.
$$

Here $n^{ \pm}=\left\{n_{1}^{ \pm}, n_{2}^{ \pm}, \ldots, n_{M}^{ \pm}\right\}$denote two increasing sequences of positive integers $1 \leq n_{1}^{+}<n_{2}^{+}<\ldots<n_{M}^{+}$and $1 \leq n_{1}^{-}<n_{2}^{-}<\ldots<n_{M}^{-}$, with $M \geq 0$; and $N_{j}\left(n^{+}\right), j=1,2, \ldots, \infty$, denotes $j$-th element of the increasing sequence of consecutive positive integers with deleted entries $n_{i}^{+}$, $i=1, \ldots M$ :

$$
\left\{N\left(n^{+}\right)\right\}=\left\{1,2, \ldots \eta_{1}^{+}, \ldots, \eta_{2}^{+}, \ldots\right\}
$$

Using the standard technique based on the Destri-de Vega equation $[10,11]$ associated with (A.2) and (A.6) one can easily calculate the next subleading term in the large $s$ asymptotics of the corresponding eigenvalues $A_{+}(s)$. The result is given by (11) with

$$
L=\sum_{i=1}^{M}\left(n_{i}^{+}+n_{i}^{-}-1\right)
$$

For a given value of $L$ there are exactly $\mathrm{p}(L)$ sets $\mathcal{S}_{k}^{(L)}=\left\{n^{+} \mid n^{-}\right\}$, with $k=1,2, \ldots \mathrm{p}(L)$, satisfying $(\mathrm{A} .8)^{5}$, where $\mathrm{p}(L)$ stands for the number of integer partitions of $L$. These sets naturally enumerate all eigenvectors in the level subspace $\mathcal{V}_{\Delta}^{(L)}{ }^{6}$. As for the set $\left\{m_{k}^{-}\right\}$describing the zeroes of $A_{-}(s)$, it is obtained from $\left\{m_{k}^{+}\right\}$by interchanging the roles of $n^{+}$and $n^{-}$in Eq.(A.6).

\footnotetext{
${ }^{5}$ The counting follows from the following elementary partition identity

$$
\sum_{n=0}^{\infty} \mathrm{p}(n) z^{n}=\sum_{n=0}^{\infty} \frac{z^{n}}{(z)_{n}}=\sum_{n=0}^{\infty} \frac{z^{n^{2}}}{(z)_{n}^{2}}, \quad(z)_{n} \equiv \prod_{k=1}^{n}\left(1-z^{k}\right) \quad(|z|<1) .
$$
}

${ }^{6}$ For example, at $L=4$, there are five sets $\mathcal{S}_{k}^{(4)},\{4 \mid 1\},\{1 \mid 4\},\{2 \mid 3\},\{3 \mid 2\},\{1,2 \mid 1,2\}$. 
As explained in [5] the formula (26) allows one to derive the asymptotic expansions for the zeroes of the eigenvalues $A_{ \pm}(s)$. Indeed, let $I_{2 n-1}$ and $\widetilde{H}_{n}^{ \pm}$ are the eigenvalues of the local IM and the dual nonlocal IM corresponding to a certain eigenvector of $\mathbf{Q}_{ \pm}(s)$ determined by the set of phases (A.6). Then substituting (26) into (A.2) one obtains

$$
\begin{aligned}
s_{k}^{\frac{1+\alpha}{2 \alpha}}+\sum_{n=1}^{\infty}\left(\nu s_{k}\right)^{\frac{(1-2 n)(1+\alpha)}{2 \alpha}} \beta_{n} I_{2 n-1}+\sum_{n=1}^{\infty}\left(\nu s_{k}\right)^{-n(1+\alpha)} \gamma_{n} \widetilde{H}_{n}^{ \pm} \\
\simeq m_{k}^{ \pm}-\frac{1}{2} \pm \frac{2 l+1}{4}
\end{aligned}
$$

where $k=1,2, \ldots$ and

$$
\begin{aligned}
& \beta_{n}=(-1)^{n} \pi^{-1} \cos (\pi(n-1 / 2) / \alpha) \quad b_{n} \\
& \gamma_{n}=(-1)^{n+1} \pi^{-1} \sin (\pi n \alpha) c_{n} .
\end{aligned}
$$

On the other hand the Bethe Ansatz equations (A.2) complemented by the asymptotic condition (A.5) can be solved numerically [12]. We have performed these calculations for the zeroes of $A_{+}(s)$ in a particular case $\alpha=4$ with various choices of the parameter $\ell$ and the integer phases $\left\{m_{k}^{+}\right\}$as in (A.6) above. For each of these choices we then calculated a few first coefficients in the asymptotic expansion (A.9) and thus determined the eigenvalues $I_{2 n-1}$ numerically (Note, that for $\alpha=4$ the second sum in (A.9) vanishes.). The results are in very good agreement with those obtained from an explicit diagonalization of the first three local IM (29). The correspondence was verified for all eigenvectors at the level $L \leq 5$.

\section{Appendix B}

Here we explain why the form (1) describes the most general potential which satisfies the conditions $(I-I I I)$ in Section 3. Indeed, let us write $V(x)$ in the form

$$
V(x)=\frac{\ell(\ell+1)}{x^{2}}+x^{2 \alpha}+v(x) .
$$

The conditions $(I-I I I)$ imply that

$$
v(x)=x^{-2} F\left(x^{2 \alpha+2}\right),
$$

where $F\left(x^{2 \alpha+2}\right)$ is a rational function of its argument, vanishing at $x=0$ and bounded at $x=\infty$,

$$
F(0)=0, \quad|F(\infty)|<\infty .
$$


Therefore, unless $F \equiv 0$, the potential (B.1) has poles at finite nonzero values of $x$. Let $x_{0} \neq 0$ be the position of any such pole. Consider the Laurent expansion of the potential (B.1) near this pole

$$
V(x)=\sum_{k=r}^{\infty}\left(x-x_{0}\right)^{k} V_{k} .
$$

It is well known (see e.g. [13]) that coefficients of this expansion should be constrained as

$$
r \geq-2 ; \quad V_{-2}=m(m+1) ; \quad V_{2 k-1}=0, \quad k=0,1, \ldots m
$$

where $m=0,1,2 \ldots$, to ensure that both linear independent solution of (12) are single valued functions $x$ in the vicinity of $x_{0}$ for all values of $E$. One can check that for $m \geq 2$ there are too many conditions in (B.5) which cannot be simultaneously satisfied for generic values of $\alpha$ and $\ell$. So the only case to consider is $m=1$ which requires

$$
V_{-2}=2, \quad V_{-1}=V_{1}=0,
$$

for every pole of (B.1). These conditions, together with (B.2) and (B.3), lead to (1) and (3).

\section{References}

[1] Voros, A. Spectral zeta functions. In: Zeta functions in geometry, eds. Kurokawa, N. and Sunada, T. Adv. Stud. in Pure Math. 21, pp. 327-358, Kinokuniya, Tokyo, 1992.

[2] Dorey, P. and Tateo, R. Anharmonic oscillators, the thermodynamic Bethe ansatz and nonlinear integral equations. J. Phys. A 32 (1999) L419-L425 [hep-th/9812211].

[3] Bazhanov, V.V., Lukyanov, S.L., and Zamolodchikov, A.B. Integrable structure of conformal field theory. II. $Q$-operator and DDV equation. Comm. Math. Phys. 190 (1997) 247-278 [hep-th/9604044].

[4] Baxter, R.J. Partition function of the eight-vertex lattice model. Ann. Phys. 70 (1972) 193-228.

[5] Bazhanov, V.V., Lukyanov, S.L., and Zamolodchikov, A.B. Spectral determinants for Schrödinger equation and Q-operators of conformal field theory. J. Stat. Phys. 102 (2001) 567-576 [hep-th/9812247]. 
[6] Bazhanov, V.V., Lukyanov, S.L., and Zamolodchikov, A.B. Integrable structure of conformal field theory, quantum KdV theory and thermodynamic Bethe Ansatz. Comm. Math. Phys. 177 (1996) 381-398 [hep-th/9412229].

[7] Affleck, I. and Ludwig, A.W.W. Critical theory of overscreened Kondo fixed points. Nucl. Phys. B 360 (1991) 641-696.

[8] Fendley, P., Saleur, H., and Warner, N.P. Exact solution of a massless scalar field with a relevant boundary interaction. Nucl. Phys. B 430 (1994) 577-596 [hep-th/9406125].

[9] Bazhanov, V.V., Lukyanov, S.L., and Zamolodchikov, A.B. Integrable structure of conformal field theory. III. The Yang-Baxter Relation. Comm. Math. Phys. 200 (1999) 297-324 [hep-th/9805008].

[10] Destri, C. and de Vega, H.J. Unified approach to thermodynamic Bethe Ansatz and finite size corrections for lattice models and field theories. Nucl. Phys. B 438 (1995) 413-454 [hep-th/9407117].

[11] Klümper, A., Batchelor, M.T., and Pearce, P.A. Central charges of the 6- and 19-vertex models with twisted boundary conditions. J. Phys. A 24 (1991) 3111-3133.

[12] Voros, A. Exact quantization condition for anharmonic oscillators (in one dimension). J. Phys. A 27 (1994) 4653-4661.

[13] Duistermaat, J.J. and Grünbaum, F.A. Differential equations in the spectral parameter. Comm. Math. Phys. 103 (1986) 177-240. 